\title{
Sequences with bounded l.c.m. of each pair of terms, III
}

\author{
by \\ Yong-Gao Chen and Li-Xia Dai (Nanjing)
}

1. Introduction. Let $A_{x}$ be a set of positive integers with the least common multiple of each pair of terms not exceeding $x$ and $\left|A_{x}\right|$ being the largest. In 1951, P. Erdős [5] (see also Guy [7]) proposed the following problem: what is the value of $\left|A_{x}\right|$ ? It is known that

$$
\sqrt{\frac{9}{8} x}+O(1) \leq\left|A_{x}\right| \leq \sqrt{4 x}+O(1) .
$$

For a proof see Erdős [6]. Choi [2] improved the upper bound to $1.638 \sqrt{x}$, and later [3] to $1.43 \sqrt{x}$. Let $B_{x}$ be the union of the set of positive integers not exceeding $\sqrt{x / 2}$ and the set of even integers between $\sqrt{x / 2}$ and $\sqrt{2 x}$. It is clear that the least common multiple of each pair of terms of $B_{x}$ does not exceed $x$. By calculation we have

$$
\left|B_{x}\right|=\sqrt{\frac{9}{8} x}+O(1) .
$$

Chen [1] gave an asymptotic formula for $\left|A_{x}\right|$ and showed that $A_{x}$ is almost the same as $B_{x}$, namely

$$
\left|A_{x} \backslash B_{x}\right|=o(\sqrt{x})
$$

In particular,

$$
\left|A_{x}\right|=\left|B_{x}\right|+o(\sqrt{x})=\sqrt{\frac{9}{8} x}+o(\sqrt{x}) .
$$

Dai and Chen [4] gave an explicit bound of the remainder for $\left|A_{x}\right|$ :

$$
\left|A_{x}\right|=\sqrt{\frac{9}{8} x}+R(x)
$$

2000 Mathematics Subject Classification: 11B75, $11 \mathrm{~B} 83$.

Key words and phrases: least common multiple, $u$-compromise, Erdős problems, distribution of primes.

Research supported by National Natural Science Foundation of China, Grant No. 10471064. The second author is supported by Science Foundation of Nanjing Normal University, Grant No. 2006101XGQ0128. 
where

$$
-2 \leq R(x) \leq \sqrt{\frac{9}{8} x}+45 \sqrt{\frac{x}{\log x}} \log \log x .
$$

On the other hand, it is natural to ask whether $R(x)=O(1)$.

Let $C_{x}$ be a set of positive integers with the least common multiple of each pair of terms not exceeding $x, B_{x} \subseteq C_{x}$ and $\left|C_{x}\right|$ being the largest. Write

$$
\left|C_{x}\right|=\left|B_{x}\right|+R_{1}(x) .
$$

If $a \in C_{x} \backslash B_{x}$, then $a \notin B_{x}$ and $[a, k] \leq x$ for all positive integers $k$ not exceeding $\sqrt{x / 2}$ and all even integers $k$ between $\sqrt{x / 2}$ and $\sqrt{2 x}$. Intuitively, this seems impossible for sufficiently large $x$. A more interesting question is whether $R_{1}(x)=O(1)$.

For any positive real number $x$ we define the function $\operatorname{loc} x$ to be the nonnegative integer $r$ with

$$
0 \leq \underbrace{\log \log \cdots \log }_{r} x<1
$$

In this paper the following results are proved.

TheOREM 1.

(i) $R_{1}(x)=0$ for infinitely many positive integers $x$;

(ii) $R_{1}(x) \geq \operatorname{loc} x-2$ for infinitely many positive integers $x$.

From Theorem 1 we have immediately

Corollary 1. $R(x) \geq \operatorname{loc} x-2$ for infinitely many positive integers $x$.

In order to study the properties of $R_{1}(x)$, we introduce the following notation.

Definition. Let $u$ be a positive real number. Two positive integers $s, t$ are $u$-compromise if there exist primes $p_{i}(i=0,1, \ldots,[u s])$ and primes $q_{j}$ $(j=0,1, \ldots,[u t])$ such that

$$
\begin{array}{ll}
p_{i} \mid s+i, & i=0,1, \ldots,[u s], \\
q_{j} \mid t+j, & j=0,1, \ldots,[u t],
\end{array}
$$

and $p_{i} \mid s-t$ when $p_{i}$ is equal to one of $q_{j}(0 \leq i \leq[u s], 0 \leq j \leq[u t])$.

It is clear that if $s, t$ are $u$-compromise, then they are also $v$-compromise for any $0<v \leq u$.

THEOREM 2. If there are three real numbers $0<u<1, \tau>0, T>0$ and a positive integer $r$ such that for any two u-compromise integers $s, t$ with $t>s \geq T$ we always have

$$
\underbrace{\log \log \cdots \log }_{r} t \geq \underbrace{\log \log \cdots \log }_{r} s+\tau,
$$


then

$$
R_{1}(x)=O(\underbrace{\log \log \cdots \log }_{r+1} x) .
$$

Corollary 2. $R_{1}(x)=O(\log \log x)$.

THEOREM 3. If there are two real numbers $0<u<1, T>0$ and a positive integer $r$ such that for any two u-compromise integers $s, t$ with $t>s \geq T$ we always have

$$
\underbrace{\log \log \cdots \log t}_{r} t \geq \frac{1}{2} \underbrace{\log \log \cdots \log }_{r-1} s,
$$

then

$$
R_{1}(x) \leq 2 \operatorname{loc} x+O(1)
$$

We pose the following problems.

Problem 1. Given any positive integer $r$, are there three real numbers $0<u<1, \tau>0$ and $T>0$ such that for any two u-compromise integers $s, t$ with $t>s \geq T$ we always have

$$
\underbrace{\log \log \cdots \log }_{r} t \geq \underbrace{\log \log \cdots \log s}_{r} s+\tau ?
$$

It is easy to prove that Problem 1 is true for $r=1$ (see the proof of Lemma 4 in the next section).

Problem 2. Are there two real numbers $0<u<1, T>0$ and a positive integer $r$ such that for any two u-compromise integers $s, t$ with $t>s \geq T$ we always have

$$
\underbrace{\log \log \cdots \log }_{r} t \geq \frac{1}{2} \underbrace{\log \log \cdots \log }_{r-1} s ?
$$

It is clear that Problem 2 is stronger than Problem 1.

\section{Proof of theorems}

LEMMA 1. Let $q$ be a prime with $3 \leq q \leq \sqrt{x / 2}$ and $4 q(q-2)>x$. Then

$$
C_{x} \subseteq\{2 l \mid l \in \mathbb{N}, l \leq x /(2 q)\} \cup\{l \mid l \in \mathbb{N}, l \leq x /(2 q), 2 \nmid l\} .
$$

Proof. Let $a \in C_{x}$. Since $q \leq \sqrt{x / 2}$, we have $q \leq x /(2 q)$. Thus we need only consider $a \neq q, 2 q$. Since $2 q, 2(q-1), 2(q-2) \in B_{x} \subseteq C_{x}$, we have

$$
[a, 2 q] \leq x, \quad[a, 2(q-1)] \leq x, \quad[a, 2(q-2)] \leq x .
$$

CAsE 1: $2 \nmid a$ and $q \nmid a$. As $2 a q=[a, 2 q] \leq x$ we have $a \leq x /(2 q)$.

CASE 2: $2 \mid a$ and $q \nmid a$. As $a q=[a, 2 q] \leq x$ we have $a / 2 \leq x /(2 q)$. 
CASe 3: $q \mid a$. Let $a=q b t$, where $t=1$ if $2 \nmid a$ and $t=2$ if $2 \mid a$. Then

$$
\begin{aligned}
& {[a, 2(q-1)]=[q b t, 2(q-1)]=2 q[b, q-1],} \\
& {[a, 2(q-2)]=[q b t, 2(q-2)]=2 q[b, q-2] .}
\end{aligned}
$$

Since $a \neq q, 2 q$, we have $b>1$. Hence either $[b, q-1] \neq q-1$ or $[b, q-2] \neq$ $q-2$. Thus

$$
\max \{[a, 2(q-1)],[a, 2(q-2)]\} \geq 4 q(q-2)>x,
$$

a contradiction. This completes the proof of Lemma 1.

LeMma 2. Let $u$ be a real number with $0<u<1, k$ be an integer with $k \leq \sqrt{x / 2}<k+1$ and $s$ be an integer such that

$$
\frac{4}{1-u}+\frac{1}{u}<s<\frac{1-u}{2 u} k
$$

and either $k+s \in C_{x}$ with $2 \nmid k+s$ or $2(k+s) \in C_{x}$. Then there exist primes $p_{i}(i=0,1, \ldots,[u s])$ such that

$$
p_{i}\left|s+i, \quad p_{i}\right| k+s, \quad i=0,1, \ldots,[u s] .
$$

Proof. Let $a=k+s$ if $k+s \in C_{x}$ with $2 \nmid k+s$, otherwise let $a=2(k+s)$. Let $i$ be an integer with $0 \leq i \leq u s$. Then $2(k-i) \in B_{x} \subseteq C_{x}$. Hence $[a, 2(k-i)] \leq x$. Since

$$
\frac{4}{1-u}+\frac{1}{u}<s<\frac{1-u}{2 u} k
$$

we have

$$
k>\frac{8 u}{(1-u)^{2}}+\frac{2}{1-u} .
$$

Hence

$$
\begin{aligned}
2(k+s)(k-i) & \geq 2(k+s)(k-u s)>2\left(k+\frac{4}{1-u}+\frac{1}{u}\right)\left(k-\frac{4 u}{1-u}-1\right) \\
& >2(k+1)^{2}>x .
\end{aligned}
$$

Noting that $[a, 2(k-i)] \leq x$ and

$$
[a, 2(k-i)]=2[k+s, k-i]=\frac{2(k+s)(k-i)}{(k+s, k-i)},
$$

we have $(k+s, k-i)>1$. Thus $(k+s, s+i)>1$. Therefore, for each $i$ with $0 \leq i \leq u s$ we may choose a prime $p_{i}$ with $p_{i} \mid k+s$ and $p_{i} \mid s+i$. This completes the proof of Lemma 2 .

Lemma 3. Let $s$ be a positive integer and $k$ be an integer with $k \leq$ $\sqrt{x / 2}<k+1$. Then $s=O(\log x)$ if $k+s \in C_{x}$ with $2 \nmid k+s$ or if $2(k+s) \in C_{x}$.

Proof. By a result on the distribution of primes and Lemma 1 we have $s=O\left(x^{\theta}\right)$, where $\theta$ is a positive constant with $\theta<1 / 2$, for example we can 
take $\theta=7 / 24$ (see Huxley [8]). Thus we may assume that $10<s<k / 2$. By Lemma 2 there exist primes $p_{i}(i=0,1, \ldots,[s / 2])$ such that

$$
p_{i}\left|s+i, \quad p_{i}\right| k+s, \quad i=0,1, \ldots,[s / 2] .
$$

Thus

$$
\prod_{s \leq p \leq 3 s / 2} p \mid k+s
$$

and so

$$
\prod_{s \leq p \leq 3 s / 2} p \leq k+s \leq x,
$$

where the product is taken over all primes $p$ in the interval $[s, 3 s / 2]$. Therefore $s=O(\log x)$. This completes the proof of Lemma 3 .

LEMMA 4. Let $k$ be an integer with $k \leq \sqrt{x / 2}<k+1$ and $s, t$ be two integers with $10<s<t<k / 2$ such that either $k+s \in C_{x}$ with $2 \nmid k+s$ or $2(k+s) \in C_{x}$, and either $k+t \in C_{x}$ with $2 \nmid k+t$ or $2(k+t) \in C_{x}$. Then $t \geq 5 s / 4$ for $s \geq M$, where $M$ is a positive constant.

Proof. By the proof of Lemma 3 we have

$$
\prod_{s \leq p \leq 3 s / 2} p\left|k+s, \quad \prod_{t \leq p \leq 3 t / 2} p\right| k+t .
$$

Hence

$$
\prod_{t \leq p \leq 3 s / 2} p \mid t-s
$$

Thus

$$
\prod_{t \leq p \leq 3 s / 2} p \leq t-s
$$

If $t<5 s / 4$, then

$$
\prod_{5 s / 4 \leq p \leq 3 s / 2} p \leq s / 4 .
$$

This cannot hold for $s$ large enough. This completes the proof of Lemma 4 .

LEMMA 5. For any positive integer $m$ we have

$$
m+\prod_{p \leq m} p \leq 2^{3 m},
$$

where the product is taken over all primes $p$ less than $m$.

Proof. We use induction on $m$. It is easy to verify the assertion for $m \leq 5$. Suppose that it is true for all positive integers less than $m$. If $m \geq 6$, then

$$
[m / 2]+1+\prod_{p \leq[m / 2]+1} p \leq 2^{3[m / 2]+3} .
$$


Since

$$
m+\prod_{[m / 2]+1<p \leq m} p \leq m+\left(\begin{array}{c}
m \\
{[m / 2]}
\end{array}\right) \leq 2^{m},
$$

we have

$$
m+\prod_{p \leq m} p \leq 2^{3[m / 2]+3+m} \leq 2^{3 m}
$$

This completes the proof of Lemma 5 .

Proof of Theorem 1

(i) Take $x=2 q^{2}$, where $q$ is an odd prime. By Lemma 1 we have $C_{x} \backslash B_{x}=\emptyset$. Hence $R_{1}(x)=0$.

(ii) Let $d_{1}=2$ and

$$
d_{n+1}=d_{n}+\prod_{p \leq 2 d_{n}-1} p, \quad n=1,2, \ldots,
$$

where the product is taken over all primes $p$ less than $2 d_{n}-1$. Then $2 \mid d_{n}$ for all $n \geq 1$. Let

$$
k_{n}=-d_{n}+\prod_{p \leq 2 d_{n}-1} p, \quad x_{n}=2 k_{n}^{2}, \quad n=1,2, \ldots
$$

By Bertrand's postulate and $2 \mid d_{n}$ we have

$$
k_{n} \geq-d_{n}+\frac{1}{2} d_{n}\left(d_{n}+1\right) \geq 3 d_{n}, \quad n \geq 2 .
$$

From (1) and $k_{1}=4, d_{1}=2, x_{1}=32$, we have $k_{n}+d_{n} \leq x_{n}(n \geq 1)$. It is clear that

$$
B_{x_{n}}=\left\{2 h \mid 1 \leq h \leq k_{n}, h \in \mathbb{Z}\right\} \cup\left\{l \mid 1 \leq l \leq k_{n}, l \in \mathbb{Z}, 2 \nmid l\right\} .
$$

Now we show that $[a, b] \leq x_{n}$ for any

$$
a, b \in B_{x_{n}} \cup\left\{2\left(k_{n}+d_{1}\right), 2\left(k_{n}+d_{2}\right), \ldots, 2\left(k_{n}+d_{n}\right)\right\} .
$$

It is clear for $n=1$. Now we assume that $n \geq 2$.

CASE 1: $a, b \in\left\{2\left(k_{n}+d_{1}\right), 2\left(k_{n}+d_{2}\right), \ldots, 2\left(k_{n}+d_{n}\right)\right\}$. Let

$$
a=2\left(k_{n}+d_{i}\right), \quad b=2\left(k_{n}+d_{j}\right) .
$$

From $2\left|d_{i}, 2\right| d_{j}, 2 \mid k_{n}$ and (1) we have

$$
[a, b] \leq\left(k_{n}+d_{i}\right)\left(k_{n}+d_{j}\right) \leq \frac{16}{9} k_{n}^{2}<x_{n} .
$$

CASE 2: $a=2\left(k_{n}+d_{i}\right)(1 \leq i \leq n)$ and $b \in B_{x_{n}}$. Without loss of generality, we may assume that $b \in\left\{2 h \mid 1 \leq h \leq k_{n}, h \in \mathbb{Z}\right\}$. Write $b=2\left(k_{n}-j\right)$.

If $j \geq d_{i}$, then $[a, b] \leq \frac{1}{2} a b \leq 2\left(k_{n}^{2}-d_{i}^{2}\right)<2 k_{n}^{2} \leq x$. 
If $0 \leq j \leq d_{i}-1$, let $p$ be a prime with $p \mid d_{i}+j$; then $p \leq 2 d_{i}-1$. Hence

$$
k_{n} \equiv-d_{n} \equiv-d_{n-1} \equiv \cdots \equiv-d_{i} \equiv j(\bmod p) .
$$

Thus

$$
(a, b)=2\left(k_{n}+d_{i}, k_{n}-j\right) \geq 2 p .
$$

By (1) and (2) we have

$$
[a, b]=\frac{a b}{(a, b)} \leq \frac{1}{2 p} a b \leq\left(k_{n}+d_{i}\right)\left(k_{n}-j\right) \leq \frac{4}{3} k_{n}^{2}<x_{n} .
$$

Therefore $[a, b] \leq x_{n}$ for any

$$
a, b \in B_{x_{n}} \cup\left\{2\left(k_{n}+d_{1}\right), 2\left(k_{n}+d_{2}\right), \ldots, 2\left(k_{n}+d_{n}\right)\right\} .
$$

To complete the proof, it is enough to prove that $n \geq \operatorname{loc} x_{n}-2$. By Lemma 5 we have $d_{i+1} \leq 2^{5 d_{i}}(i \geq 1)$. Thus $\log d_{i+1} \leq 5 d_{i}(i \geq 1)$. Hence

$$
\begin{gathered}
\log x_{n}=\log 2+2 \log k_{n} \leq \log 2+2 \log d_{n+1} \leq 11 d_{n}, \\
\log \log x_{n} \leq \log 11+\log d_{n} \leq 7 d_{n-1} .
\end{gathered}
$$

Continuing this procedure, we have

$$
\underbrace{\log \log \cdots \log }_{i} x_{n} \leq 7 d_{n+1-i}
$$

Since $\operatorname{loc}\left(7 d_{1}\right)=2$, we have $\operatorname{loc} x_{n} \leq n+2$. This completes the proof of Theorem 1.

Proof of Theorem 2. Assume that $x$ is large enough. Without loss of generality, we may assume that

$$
\underbrace{\log \log \cdots \log T}_{r} T>0 \text {. }
$$

Let $k$ be an integer with $k \leq \sqrt{x / 2}<k+1$ and let $t_{1}, \ldots, t_{l}$ be positive integers with

$$
\max \left\{T, \frac{4}{1-u}+\frac{1}{u}\right\}<t_{1}<\cdots<t_{l}
$$

and either $k+t_{i} \in C_{x}$ with $2 \nmid k+t_{i}$ or $2\left(k+t_{i}\right) \in C_{x}(1 \leq i \leq l)$. By Lemma 3 we have $t_{l}=O(\log x)$. Hence we may assume that $t_{l}<(1-u) k /(2 u)$. By Lemma 2 and the definition of $u$-compromise we see that $t_{i}, t_{i+1}$ are $u$-compromise $(1 \leq i \leq l-1)$. Hence

$$
\underbrace{\log \log \cdots \log }_{r} t_{i+1} \geq \underbrace{\log \log \cdots \log }_{r} t_{i}+\tau, \quad 1 \leq i \leq l-1 .
$$

Thus

$$
\underbrace{\log \log \cdots \log }_{r} t_{l} \geq \underbrace{\log \log \cdots \log }_{r} t_{1}+(l-1) \tau \geq(l-1) \tau .
$$


Noting that $t_{l}=O(\log x)$, we have

$$
l=O(\underbrace{\log \log \cdots \log x}_{r+1}) .
$$

Therefore

$$
R_{1}(x)=O(\underbrace{\log \log \cdots \log x}_{r+1}) .
$$

This completes the proof of Theorem 2 .

Corollary 2 follows from Lemma 4 and Theorem 2 immediately.

Proof of Theorem 3. The initial part is as in the proof of Theorem 2. Then

$$
\underbrace{\log \log \cdots \log }_{r} t_{i+1} \geq \frac{1}{2} \underbrace{\log \log \cdots \log }_{r-1} t_{i}, \quad 1 \leq i \leq l-1 .
$$

Without loss of generality, we may assume that

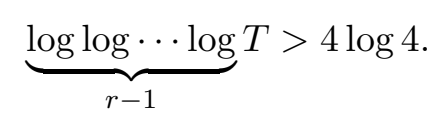

Thus

$$
\underbrace{\log \log \cdots \log }_{r-1} t_{i}>4 \log 4, \quad 1 \leq i \leq l
$$

Hence

$$
\begin{aligned}
\underbrace{\log \log \cdots \log }_{r+1} t_{l} & \geq \log \frac{1}{2}+\underbrace{\log \log \cdots \log }_{r} t_{l-1} \\
& \geq \log \frac{1}{2}+\frac{1}{2} \underbrace{\log \log \cdots \log }_{r-1} t_{l-2} \\
& \geq \frac{1}{4} \underbrace{\log \log \cdots \log }_{r-1} t_{l-2} .
\end{aligned}
$$

Continuing this procedure we have

$$
\underbrace{\log \log \cdots \log }_{r+l-2} t_{l} \geq \frac{1}{4} \underbrace{\log \log \cdots \log }_{r-1} t_{1} \geq \frac{1}{4} \underbrace{\log \log \cdots \log }_{r-1} T \geq 1 .
$$

Hence loc $t_{l} \geq r+l-2$. Since $t_{l} \leq x$, we have

$$
\operatorname{loc} x \geq r+l-2 \text {. }
$$

Therefore

$$
R_{1}(x) \leq 2 l+O(1) \leq 2 \operatorname{loc} x+O(1) .
$$




\section{References}

[1] Y. G. Chen, Sequences with bounded l.c.m. of each pair of terms, Acta Arith. 84 (1998), 71-95.

[2] S. L. G. Choi, The largest subset in $[1, n]$ whose integers have pairwise l.c.m. not exceeding n, Mathematika 19 (1972), 221-230.

[3] - , The largest subset in $[1, n]$ whose integers have pairwise l.c.m. not exceeding $n$, II, Acta Arith. 29 (1976), 105-111.

[4] L. X. Dai and Y. G. Chen, Sequences with bounded l.c.m. of each pair of terms, II, ibid. 124 (2006), 315-326.

[5] P. Erdős, Problem, Mat. Lapok 2 (1951), 233.

[6] - Extremal problems in number theory, in: Theory of Numbers, Proc. Sympos. Pure Math. 8, Amer. Math. Soc., 1965, 181-189.

[7] R. K. Guy, Unsolved Problems in Number Theory, 2nd ed., Springer, New York, 1994.

[8] M. N. Huxley, On the difference between consecutive primes, Invent. Math. 15 (1972), 164-170.

Department of Mathematics

Nanjing Normal University

Nanjing 210097, Jiangsu

P.R. China

E-mail: ygchen@njnu.edu.cn

Received on 25.9.2006

and in revised form on 21.12.2006 